\title{
Relation between current sheets and vortex sheets in stationary incompressible MHD
}

\author{
D. H. Nickeler ${ }^{1,2}$ and T. Wiegelmann ${ }^{2}$ \\ ${ }^{1}$ Astronomical Institute AV ČR Ondřejov, Fričova 298, 25165 Ondrejov, Czech Republic \\ ${ }^{2}$ Max-Planck-Institut für Sonnensystemforschung, Max-Planck-Strasse 2, 37191 Katlenburg-Lindau, Germany \\ Correspondence to: D. H. Nickeler (nickeler@asu.cas.cz)
}

Received: 14 September 2011 - Revised: 21 December 2011 - Accepted: 10 February 2012 - Published: 16 March 2012

\begin{abstract}
Magnetohydrodynamic configurations with strong localized current concentrations and vortices play an important role in the dissipation of energy in space and astrophysical plasma. Within this work we investigate the relation between current sheets and vortex sheets in incompressible, stationary equilibria. For this approach it is helpful that the similar mathematical structure of magnetohydrostatics and stationary incompressible hydrodynamics allows us to transform static equilibria into stationary ones. The main control function for such a transformation is the profile of the Alfvén-Mach number $M_{\mathrm{A}}$, which is always constant along magnetic field lines, but can change from one field line to another. In the case of a global constant $M_{\mathrm{A}}$, vortices and electric current concentrations are parallel. More interesting is the nonlinear case, where $M_{\mathrm{A}}$ varies perpendicular to the field lines. This is a typical situation at boundary layers like the magnetopause, heliopause, the solar wind flowing around helmet streamers and at the boundary of solar coronal holes. The corresponding current and vortex sheets show in some cases also an alignment, but not in every case. For special density distributions in 2-D, it is possible to have current but no vortex sheets. In 2-D, vortex sheets of field aligned-flows can also exist without strong current sheets, taking the limit of small Alfvén Mach numbers into account. The current sheet can vanish if the Alfvén Mach number is (almost) constant and the density gradient is large across some boundary layer. It should be emphasized that the used theory is not only valid for small Alfvén Mach numbers $M_{\mathrm{A}} \ll 1$, but also for $M_{\mathrm{A}} \lesssim 1$. Connection to other theoretical approaches and observations and physical effects in space plasmas are presented. Differences in the various aspects of theoretical investigations of current sheets and vortex sheets are given.
\end{abstract}

Keywords. Space plasma physics (Kinetic and MHD theory)

\section{Introduction}

Many structures in magnetospheres or in the solar corona are often described by quasi-magnetohydrostatic sequences of equilibria (e.g. Romeou and Neukirch, 1999; Schindler and Birn, 1982; Becker et al., 2001; or Wiegelmann and Schindler, 1995). Current sheets are important for storage and release, i.e. dissipation of energy, leading, e.g. to magnetic reconnection (see Neukirch, 2005b, or Solanki et al., 2003 , for observational aspects of coronal heating). The formation of thin current sheets is often done within the frame of the quasi-static approach, where the time dependent pressure on the boundary is prescribed. Via the law of adiabatic change of the flux tubes in the frame of ideal MHD magnetospheric convection, the flux transport takes place and forms highly structured current sheets.

Another approach to the formation of current sheets is a parametric method (e.g. Nickeler et al., 2006, and Nickeler and Wiegelmann, 2010). Here the parameter is the Alfvén Mach number instead of the control parameters (e.g. Forbes and Priest, 1995, and Romeou and Neukirch, 1999). These parameters describe constraints or boundary conditions, like magnetic shear, pressure or currents.

Instabilities of current and vortex sheets, although with very high Alfvén Mach number, have been analysed in Baty et al. (2003). In this article, grid-adaptive simulations show that even in very weak magnetic field regimes $\left(M_{\mathrm{A}} \simeq 30\right)$, the large-scale Kelvin-Helmholtz coalescence process can trigger tearing-type reconnection events previously identified in cospatial current-vortex sheets.

The paper of Eyink and Aluie (2006) explains how, for discontinuous fields, a part of the magnetic flux can slip through the plasma by an averaged turbulent statistic fluctuation of a subscale electromotive force (EMF), i.e. an electric field in the comoving systems of the ions. Another important aspect 
of this paper is that current sheets and vortex sheets both have to exist and "intersect in sets of large enough dimensions" to get "ideal" magnetic reconnection, i.e. "magnetic flux conservation may be broken in ideal MHD by nonlinear effects". Such tangential discontinuities are basically current vortex sheets.

From numerical, incompressible, time-dependent, adaptive MHD-simulations by Grauer and Marliani (1998, 2000), it is shown that vortex sheets align with current sheets. The initial conditions in this investigation are given by the socalled Orszag-Tang vortex.

In the present paper, we analyse and explain some of the physical implications of the non-canonical transformation technique (as developed and discussed by, e.g. Gebhardt and Kiessling, 1992; Petrie and Neukirch, 1999) to calculate steady-state ideal MHD flows with current sheets. We analyse the feedback circuits on magnetohydrodynamic forces that arise, enabling a relaxed, incompressible steady-state plasma flow along a magnetohydrostatic background field. We analyse the connection between vortex and current sheets in the frame of this mathematical technique, but also for general field-aligned flows.

In Sect. 2 we discuss the basic stationary MHD equations and introduce a general solution for the incompressible field aligned case, based on magnetohydrostatic equilibria. In particular, we investigate the forces induced by the flow. Section 3 contains special cases of initial potential and forcefree equilibria. In Sect. 4 we investigate the relation between electric currents and flow vortices in general and provide analytic example solutions in Sect. 5. Finally, we discuss the impact of this work on physical objects like planetary magnetospheres and the solar coronal plasma.

\section{Transformation from magnetohydrostatic equilibria to stationary MHD configurations}

Under the assumption of a finite Alfvén Mach number, the equations of stationary incompressible and field-aligned MHD are given by

$$
\begin{aligned}
\nabla \cdot \boldsymbol{v} & =0, \quad \boldsymbol{v} \cdot \nabla \rho=0, \\
\rho(\boldsymbol{v} \cdot \nabla) \boldsymbol{v} & =\boldsymbol{j} \times \boldsymbol{B}-\nabla p, \\
\boldsymbol{v} & =\frac{M_{\mathrm{A}} \boldsymbol{B}}{\sqrt{\mu_{0} \rho}}, \\
\nabla \cdot \boldsymbol{B} & =0,
\end{aligned}
$$

where $\boldsymbol{v}$ is the velocity, $\rho$ the mass density, $\boldsymbol{j}=\frac{1}{\mu_{0}} \nabla \times \boldsymbol{B}$ the current density vector, $p$ the scalar plasma pressure, and $M_{\mathrm{A}}$ is the Alfvén Mach number. The assumption that the magnetic field and the flow are parallel leads to the conclusion that the electric field has to vanish everywhere identically to fulfill ideal Ohm's law.

Within this work we solve the equations by a transformation approach developed in Gebhardt and Kiessling (1992), which transforms a static solution into a stationary one. We show that the general solutions of the stationary, field-aligned incompressible ideal MHD equations lead to a very close connection between current and vortex sheets. We also show how the forces are compensated by the exact solution method used.

In the following, we transform the force terms to enlighten the connection to the magnetic and plasma force terms. Under the assumption of field aligned stationary incompressible plasma flow, we get the transformation equations (see Nickeler and Wiegelmann, 2010; Nickeler et al., 2006, for details). Keeping in mind that the mass continuity Eqs. (1) and (4) for every Alfvén Mach number $M_{\mathrm{A}}$ and the density $\rho$ satisfying $\boldsymbol{B} \cdot \nabla M_{\mathrm{A}}=0$ and $\boldsymbol{B} \cdot \nabla \rho=0$ is a necessary condition, the general solution of the system Eqs. (1)-(4) in 3-D, but also in 2-D, is given by

$$
\begin{aligned}
\boldsymbol{B} & =\frac{\boldsymbol{B}_{S}}{\sqrt{1-M_{\mathrm{A}}^{2}}}, \\
p & =p_{S}-\frac{1}{2 \mu_{0}} \frac{M_{\mathrm{A}}^{2}\left|\boldsymbol{B}_{S}\right|^{2}}{1-M_{\mathrm{A}}^{2}}, \\
\sqrt{\rho} \boldsymbol{v} & =\frac{1}{\sqrt{\mu_{0}}} \frac{M_{\mathrm{A}} \boldsymbol{B}_{S}}{\sqrt{1-M_{\mathrm{A}}^{2}}}, \\
\boldsymbol{j} & =\frac{M_{\mathrm{A}}}{\mu_{0}} \frac{\nabla M_{\mathrm{A}} \times \boldsymbol{B}_{S}}{\left(1-M_{\mathrm{A}}^{2}\right)^{\frac{3}{2}}}+\frac{\boldsymbol{j}_{S}}{\left(1-M_{\mathrm{A}}^{2}\right)^{\frac{1}{2}}}, \\
\nabla p_{S} & =\boldsymbol{j}_{S} \times \boldsymbol{B}_{S},
\end{aligned}
$$

where the subscript $S$ defines the original magnetohydrostatic fields.

Thus, one has a recipe to construct field-aligned, incompressible flows along magnetohydrostatic structures: the first step is to find a magnetohydrostatic (MHS) equilibrium, which we label with the subscript $S$ for static $\left(p_{S}, \boldsymbol{B}_{S}\right)$. For the mathematical method introduced by Gebhardt and Kiessling (1992), the static equilibria is written with the help of Euler potentials $(\alpha, \beta): \boldsymbol{B}=\nabla \alpha \times \nabla \beta$ or if the equilibrium has some sort of symmetry (e.g. in z-direction) with the flux function $A: \boldsymbol{B}=\nabla A(x, y) \times \boldsymbol{e}_{\mathrm{z}}+B_{\mathrm{Z}}(x, y) \boldsymbol{e}_{\mathrm{z}}$. The next step is to specify a Mach number profile $M_{\mathrm{A}}(\alpha, \beta)$, or $M_{\mathrm{A}}(A)$ in 2$\mathrm{D}$, depending on the Euler potentials or flux function, respectively. One should be aware that the function $\rho$ as a function of the two Euler-potential is independent of the choice of the Alfvén Mach number. They are basically two independent functions.

The analysis of the forces deals with all the force terms in the Euler-equation of ideal MHD, namely

$\rho(\boldsymbol{v} \cdot \nabla) \boldsymbol{v}=\boldsymbol{j} \times \boldsymbol{B}-\nabla p$.

The identities Eqs. (5)-(7) lead with the Eqs. (1)-(4) to

$\rho(\boldsymbol{v} \cdot \nabla) \boldsymbol{v}=\frac{M_{\mathrm{A}}^{2}}{\mu_{0}\left(1-M_{\mathrm{A}}^{2}\right)}\left(\boldsymbol{B}_{s} \cdot \nabla\right) \boldsymbol{B}_{s}$. 
This means that the new inertial force, induced by the flow, depends mainly on the magnetic tension of the magnetohydrostatic field, and of course on a function of the Alfvén Mach number, i.e. the inertial force is a transformed part of the magnetic tension force of the magnetohydrostatic field.

We are now going to analyse the right hand side of the momentum or force equation Eq. (10), where we can identify the Lorentz force by

$j \times B=f_{\mathrm{L} 1}+f_{\mathrm{L} 2}$,

where

$\boldsymbol{f}_{\mathrm{L} 1}=\frac{\boldsymbol{j}_{s} \times \boldsymbol{B}_{s}}{1-M_{\mathrm{A}}^{2}}$

$f_{\mathrm{L} 2}=-\frac{1}{\mu_{0}} \frac{M_{\mathrm{A}} B_{s}^{2}}{\left(1-M_{\mathrm{A}}^{2}\right)^{2}} \nabla M_{\mathrm{A}}$.

Here we recognize that the initial Lorentz-force is enhanced by a factor $1 /\left(1-M_{\mathrm{A}}^{2}\right)$ and changed by a term depending on the gradient of the Alfvénic Mach number and the magnetic pressure. But the the term with gradient of $M_{\mathrm{A}}$ is also influenced strongly by the strength of the Alfvén Mach number itself. If $M_{\mathrm{A}} \lesssim 1$ the term with the gradient of $M_{\mathrm{A}}$ is enhanced by the $\left(1-M_{\mathrm{A}}^{2}\right)^{2}$-term, having, of course, even a stronger influence than the $\left(1-M_{\mathrm{A}}^{2}\right)$-term in the nominator of the modified original Lorentz-force $f_{\mathrm{L} 1}$.

The second term on the right hand side of the momentum Eq. (10) is given by the pressure force $\nabla p_{s}$ transformed via

$$
\begin{aligned}
-\nabla p= & -\nabla\left(p_{S}-\frac{1}{2 \mu_{0}} \frac{M_{\mathrm{A}}^{2} \boldsymbol{B}_{s}^{2}}{1-M_{\mathrm{A}}^{2}}\right) \\
= & -\nabla p_{S} \\
& -\frac{M_{\mathrm{A}}^{2}}{1-M_{\mathrm{A}}^{2}} j_{S} \times \boldsymbol{B}_{S} \\
& +\frac{1}{2 \mu_{0}} B_{S}^{2} \nabla \frac{M_{\mathrm{A}}^{2}}{1-M_{\mathrm{A}}^{2}} \\
& +\frac{M_{\mathrm{A}}^{2}}{\mu_{0}\left(1-M_{\mathrm{A}}^{2}\right)}\left(\boldsymbol{B}_{s} \cdot \nabla\right) \boldsymbol{B}_{s} .
\end{aligned}
$$

Thus, after final addition of the new pressure gradient to the new Lorentz-force on the right hand side of the momentum equation Eq. (10), the only remaining force-term is the net force, namely the modified magnetohydrostatic tension force

$\frac{1}{\mu_{0}} M_{\mathrm{A}}^{2} /\left(1-M_{\mathrm{A}}^{2}\right)\left(\boldsymbol{B}_{S} \cdot \nabla\right) \boldsymbol{B}_{S} \equiv \rho(\boldsymbol{v} \cdot \nabla) \boldsymbol{v}$.

The remaining term

$\nabla p_{S}-\boldsymbol{j}_{S} \times \boldsymbol{B}_{S}$

vanishes, because this describes the original static equilibrium. As it can be recognized by the inertial force (=tension force) in Eq. (17), the expression can become extremely large in the limit of $M_{\mathrm{A}} \rightarrow 1$.
This implies that the modified pressure gradient consists of four terms: the first is the static pressure, the second a term completing the new Lorentz force to the old static one, the third term removes the gradient forces concerning the Mach number gradient term in Eq. (12), and the fourth term displays the final inertia or net force term of the flow, induced by the mapping method.

\subsection{Comparison between quasi-static and stationary approach}

If the gradient of $M_{\mathrm{A}}$ is very large and also $M_{\mathrm{A}}$ has a value close to one, as explained in the last section and following the last paragraph of the Lorentz-force Eq. (12), hence the complete Lorentz-force can become extremely large. The situation in the quasi-static approach is different: Although the current density grows extremely in the forming thin current sheet, no strong Lorentz-forces occur due to the null sheet of the magnetic field, ignoring a small normal component to the sheet.

In the case of the stationary states even for flows with small or moderate Alfvén Mach numbers, the magnetic flux density and therefore the magnetic flux is not essentially enhanced, compared to the initial MHS state, but the newly generated current density is able to increase in order of magnitude. Additionally, filamentary or "fractal" structures, compared to the old magnetohydrostatic current distribution, are created, i.e. a double-structure evolves (see (Nickeler and Wiegelmann, 2010), and also see Figs. 1, 2). The pressure gradient is enhanced and has to be compensated by the Lorentz-force as the additional inertial force, due to the flow is of second order in $M_{\mathrm{A}}$, see Eq. (11) for small $M_{\mathrm{A}} \ll 1$, and therefore negligible, but not negligible for large $M_{\mathrm{A}}$. The only price one has to pay here is the unchanged shape of the fieldlines, in contrast to the quasi-static approach, and the formation of strong shear flows, i.e. small scale vortices, as we will show in the next sections.

The additionally occuring filamentary fine structures of the steady-state current sheets, i.e. thin current sheets, are therefore similar to results found, e.g. in Wiegelmann and Schindler (1995). These authors found that only for nonsimilarity solutions of the quasi-static equations doublestructure of the current density evolves. The evolution of a double-structure is necessary for the occurence of thin current sheets.

However, the method of thin current sheet formation and structure used in this paper is a different approach than the quasi-static approach (e.g. see Schindler and Birn, 1982, or Wiegelmann and Schindler, 1995), where the pressure is applied as boundary condition and via the law of adiabatic change is equivalent to magnetic flux transport and therefore flux enhancement. This is in contrast to the approach of this paper, where at least for small Alfvén Mach numbers the flux is not essentially enhanced. 

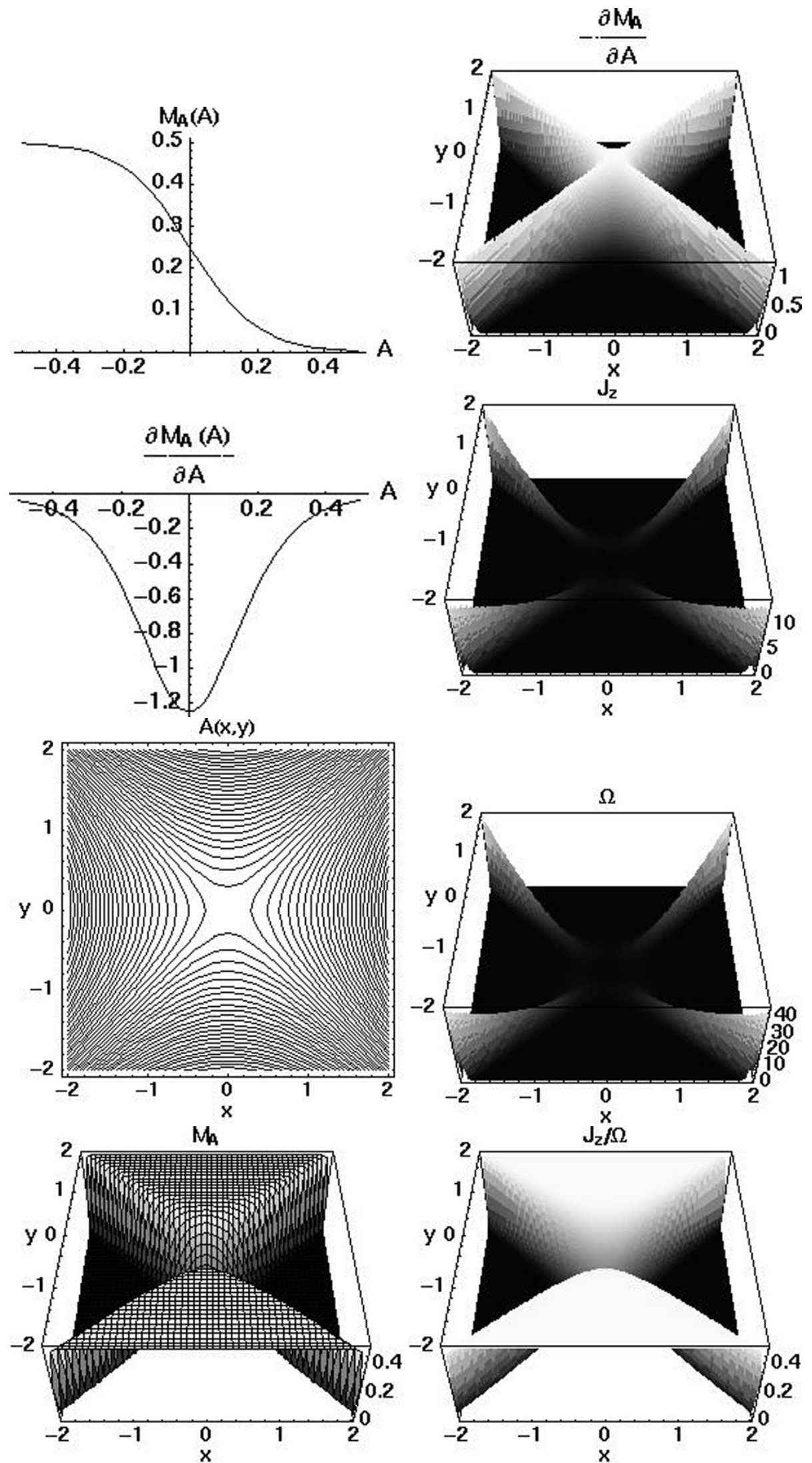

Fig. 1. Transformation from an initial potential field with a smooth Mach-number profile (see Eq. 37 with $M_{\text {Amax }}=0.5$ and $d=0.2$ ). 

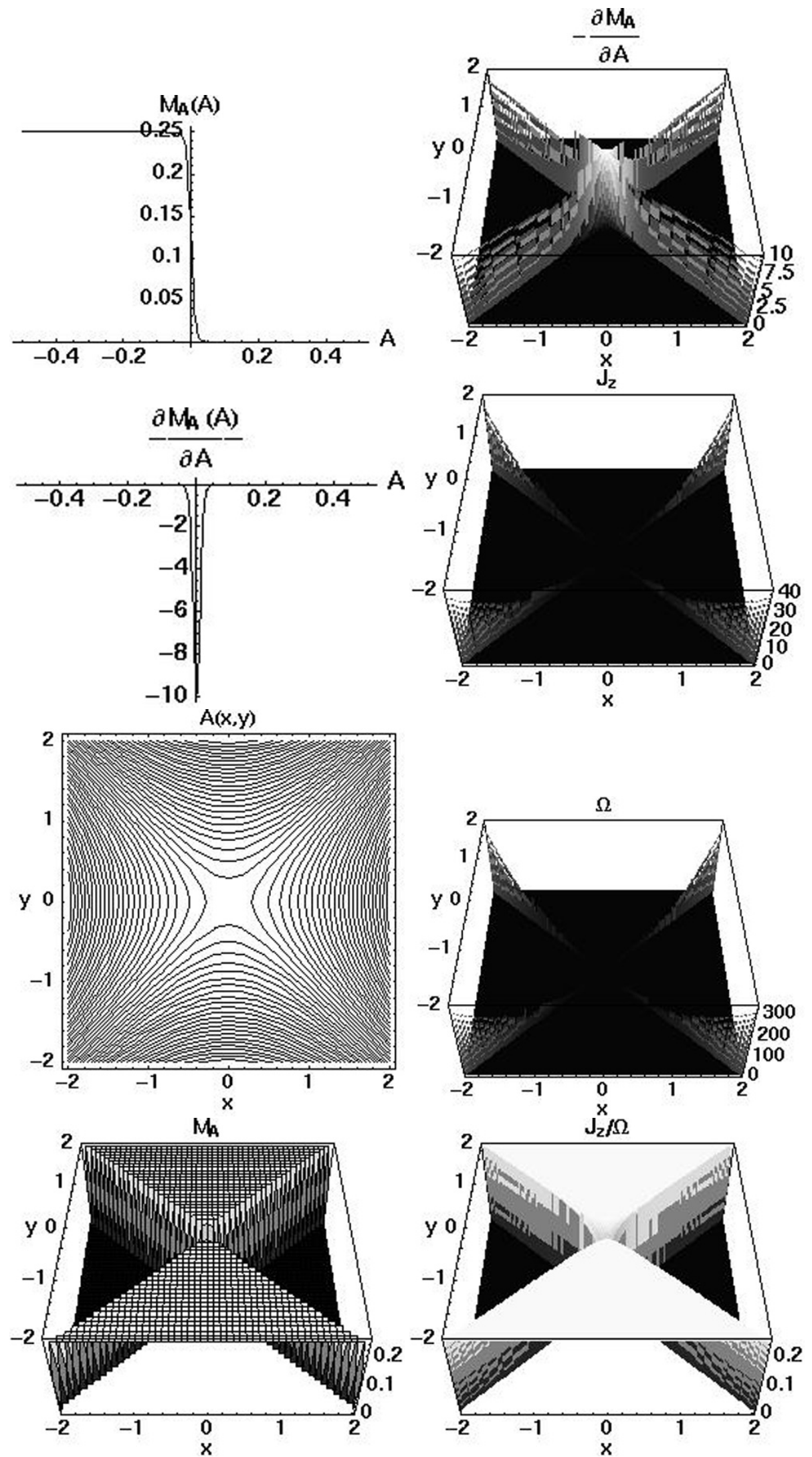

Fig. 2. Transformation from an initial potential field, similar as in Fig. 1, but with a much steeper Mach-number profile (see Eq. 37 with $M_{\text {Amax }}=0.25$ and $\left.d=0.2 / 16\right)$. 
One interesting aspect of the velocity fields, as explained and calculated in Wiechen and Schindler (1988) and Schindler and Birn (1987), is that the velocity component parallel to the magnetic field in the vicinity of separatrices is getting very large. Hence also in the quasi-static regime, the flow parallel to the magnetic field is of huge importance for the plasma dynamics.

\section{Special cases}

To see how the transformation technique affects the initial MHS-equilibrium we will now discuss and analyse some properties of specially chosen MHS-equilibria and flows, i.e. Alfvén Mach numbers.

\subsection{Flows with global constant Alfvén Mach number}

\subsubsection{Potential field as initial static equilibrium}

The most simple cases of field-aligned incompressible MHD flows seem to be represented by potential fields as initial MHS-fields and the restriction to constant Alfvén Mach numbers. We now analyse some combinations of these possibilities. Discussing $j_{s}=0$ and $M_{\mathrm{A}}=$ const it can clearly be recognized in the modified Lorentz force Eq. (12) that the magnetic field is similar to the old magnetohydrostatic field, i.e. the static field does not change its relative field strength across the field lines. The magnetic field stays potential, no currents form. The gradient forces vanishes, i.e. $\nabla p_{S}=\mathbf{0}$, there is no Lorentz-force, and only the constant static pressure $p_{S}$ changes to an inverted shaped thermal pressure $p$, reduced by a fraction of the magnetic pressure, depending on $M_{\mathrm{A}}$. One needs large enough offsets in that case, to avoid negative thermal pressures $p$. The net force or inertial force from the flow can then be interpreted as the gradient force of the plasma pressure in the magnetohydrostatic case times $M_{\mathrm{A}}^{2} /\left(1-M_{\mathrm{A}}^{2}\right)$, which for $\boldsymbol{\nabla} \times \boldsymbol{B}_{S}=0$ is identical to the magnetic tension force. Without gradients of $M_{\mathrm{A}}$ no additional forces can be generated.

\subsubsection{Initial force-free fields}

The Lorentz-force equation Eq. (12) shows also clearly that instead of the initially force-free state a force is induced by the flow gradient term $\nabla M_{\mathrm{A}}$. All other aspects are similar to the potential field case.

\subsubsection{Non force-free initial magnetohydrostatic equilibria}

With this trivial case that $j_{s} \neq 0$ and $M_{\mathrm{A}}=$ const, we get solutions very similar to the initial MHS equilibria. The magnetic field and the current are mapped by a constant factor $1 / \sqrt{1-M_{\mathrm{A}}^{2}}$, and therefore the Lorentz force is enhanced by the constant factor $1 /\left(1-M_{\mathrm{A}}^{2}\right)$. The current sheet structure is conserved, and therefore basically these solutions represent similarity solutions, as all terms, including gradients of the Mach number, vanish.

\subsection{Flows with non-constant Alfvén Mach number profile}

The generic case of plasma flows is that the Mach number changes from one field line to the other and the transformation equations are non-linear. This leads to additional nonlinear effects as discussed in the following.

\subsubsection{Initial potential field}

The special case of $j_{s}=0$ and $M_{\mathrm{A}} \neq$ const is simple, but very interesting for easy modelling: one can choose a potential, e.g. asymptotical homogeneous field and map it with multiple current sheets, as done in Nickeler et al. (2006). The effect on the forces is that the Bernoulli pressure force within the new pressure force, i.e. $-\nabla p=-\nabla\left(p_{S}-\rho v^{2} / 2\right)$, must be compensated by an additional Lorentz force. As the $f_{\mathrm{L} 1-}$ term in Eq. (12) is zero, as $j_{S}=0$, only the force-term $\boldsymbol{f}_{\mathrm{L} 1}$ in Eq. (12) can do that job. The reason is that only the gradient of the Alfvén Mach number can generate currents and then induce additional Lorentz forces. The net force or inertial force originating from the flow can then be interpreted as the gradient force of the magnetohydrostatic pressure times $M_{\mathrm{A}}^{2} /\left(1-M_{\mathrm{A}}^{2}\right)$. The fact that $M_{\mathrm{A}}$ is non-constant produces currents perpendicular to the magnetic field, repeating and keeping in mind that,

$$
\begin{gathered}
\mu_{0} \boldsymbol{j}=M_{\mathrm{A}} \frac{\nabla M_{\mathrm{A}} \times \boldsymbol{B}_{S}}{\left(1-M_{\mathrm{A}}^{2}\right)^{3 / 2}}+\frac{\nabla \times \boldsymbol{B}_{S}}{\left(1-M_{\mathrm{A}}^{2}\right)^{1 / 2}} \\
\stackrel{\text { here }}{=} M_{\mathrm{A}} \frac{\nabla M_{\mathrm{A}} \times \boldsymbol{B}_{S}}{\left(1-M_{\mathrm{A}}^{2}\right)^{3 / 2}} .
\end{gathered}
$$

The equation reflects that in this case the generated currents are perpendicular to the magnetic field but also to the direction in which the flow gradient is oriented.

\subsubsection{Initial force-free fields}

While the first term in the first line of Eq. (19) provides only perpendicular currents with respect to the magnetic field, if the Alfvén Mach number has strong gradients, the effect of the second term is only to enhance the existing magnetohydrostatic force-free current, being parallel to the magnetic field, written as

$$
\begin{aligned}
\mu_{0} \boldsymbol{j} & =M_{\mathrm{A}} \frac{\nabla M_{\mathrm{A}} \times \boldsymbol{B}_{S}}{\left(1-M_{\mathrm{A}}^{2}\right)^{3 / 2}}+\frac{\nabla \times \boldsymbol{B}_{S}}{\left(1-M_{\mathrm{A}}^{2}\right)^{1 / 2}} \\
& =M_{\mathrm{A}} \frac{\nabla M_{\mathrm{A}} \times \boldsymbol{B}_{S}}{\left(1-M_{\mathrm{A}}^{2}\right)^{3 / 2}}+\frac{\alpha \boldsymbol{B}_{S}}{\left(1-M_{\mathrm{A}}^{2}\right)^{1 / 2}},
\end{aligned}
$$

where $\nabla \times \boldsymbol{B}_{S}=\alpha \boldsymbol{B}_{S}$. This implies that a former force-free field without flows now develops components of the current, 
producing forces pointing into the direction of $\nabla M_{\mathrm{A}}$ (see modified Lorentz-force in Eq. (12)).

\section{Connection between vortices and currents in field- aligned, subalfvénic, stationary ideal MHD}

Even if the notions of "turbulent" and "ideal" reconnection (see, e.g. (Eyink and Aluie, 2006) or other references), are based on discontinuous solutions, also including a specific angle between vortex sheet and current sheet, the solutions presented here represent a powerful method to find the limit from continuous, namely well-known MHS solutions with smooth current sheets, to almost discontinuous solutions, i.e. sufficiently small subscales of concentrated and strong current sheets. It is obvious that for $\boldsymbol{v} \| \boldsymbol{B}$, the "curls" of both vector fields, i.e. their vortices, are directly connected, of course not necessarily parallel ${ }^{1}$

$\boldsymbol{v}=\frac{M_{\mathrm{A}} \boldsymbol{B}}{\sqrt{\mu_{0} \rho}}:=\lambda \boldsymbol{B} \Rightarrow \nabla \times \boldsymbol{v}=\nabla \lambda \times \boldsymbol{B}+\lambda \boldsymbol{\nabla} \times \boldsymbol{B}$.

As $\boldsymbol{\nabla} \times \boldsymbol{B}$ can be split into one perpendicular component $\boldsymbol{j}_{\perp}$ with respect to the magnetic field and one parallel component, $\boldsymbol{j}_{\|}$, we can rewrite the curl of the velocity

$\nabla \times \boldsymbol{v}=\nabla \lambda \times B+\frac{\lambda}{\mu_{0}}\left(j_{\|}+j_{\perp}\right)$.

This implies that

$$
\boldsymbol{B} \cdot(\boldsymbol{\nabla} \times \boldsymbol{B})=0 \quad \Rightarrow \quad \boldsymbol{j}_{\|}=\mathbf{0}
$$

is a necessary condition for a MHD configuration with a nonvanishing current but without vortices in 3-D (or 2-D). This is necessary as the term with the gradient of $\lambda$, being perpendicular to the magnetic field, cannot compensate the term with $\boldsymbol{j}_{\|}$. For a 2.5-D configuration, the parallel part of the current can only vanish identically if everywhere $(\nabla A)^{2}$ can be expressed as a function of $A$. Thus, in a physical situation with currents, but without vortices, it is necessary that the parallel current vanishes. We will present a sufficient criterion in 2-D (Eq. 25) as an example.

With the additional assumptions that the flow is incompressible, we can simplify the relations (21), getting the vorticity or vortex (strength) $\boldsymbol{\Omega}$,

$$
\begin{aligned}
\boldsymbol{\Omega}= & \nabla \times\left(\frac{M_{\mathrm{A}} \boldsymbol{B}}{\sqrt{\mu_{0} \rho}}\right)=-\frac{M_{\mathrm{A}} \nabla \rho \times \boldsymbol{B}}{2\left(\mu_{0} \rho^{3}\right)^{1 / 2}}+\frac{\nabla \times\left(M_{\mathrm{A}} \boldsymbol{B}\right)}{\sqrt{\rho}} \\
= & \frac{M_{\mathrm{A}} \boldsymbol{B}_{S} \times \nabla \rho}{2 \sqrt{\mu_{0}}\left(1-M_{\mathrm{A}}^{2}\right)^{1 / 2} \rho^{3 / 2}}+\frac{\nabla M_{\mathrm{A}} \times \boldsymbol{B}_{S}}{\sqrt{\mu_{0} \rho}\left(1-M_{\mathrm{A}}^{2}\right)^{3 / 2}} \\
& +\frac{M_{\mathrm{A}} \nabla \times \boldsymbol{B}_{S}}{\sqrt{\mu_{0} \rho}\left(1-M_{\mathrm{A}}^{2}\right)^{1 / 2}} .
\end{aligned}
$$

For potential fields $\left(\boldsymbol{\nabla} \times \boldsymbol{B}_{S}=\mathbf{0}\right)$, the vorticity only has components perpendicular to the magnetic field; if $M_{\mathrm{A}}$ is constant, then the vorticity is only non-zero, if the density is not

\footnotetext{
${ }^{1}$ For global constant $M_{\mathrm{A}} / \sqrt{\rho}$ they are strictly parallel.
}

completely constant. Strong vortex sheets only occur if the density gradient is strong enough.

In the case of force-free fields, there are nonvanishing components of the vorticity, even if $M_{\mathrm{A}}$ is constant, while the current remains field-aligned. The second term in the last equation also enables generation of a component of the vorticity perpendicular to the magnetic field in the case $\nabla M_{\mathrm{A}} \neq \mathbf{0}$, but it is, of course, also perpendicular to the current.

In the nonlinear case with small $M_{\mathrm{A}}=$ const, the vortex can be large, while the current is not extremely enhanced by the flow. Necessary for $\boldsymbol{\Omega}=\mathbf{0}$, e.g. in 2-D with $\boldsymbol{\nabla} \times \boldsymbol{B}_{S}=\mathbf{0}$ is, with apostrophes denoting derivatives with respect to the flux function $A$,

$$
\begin{aligned}
& \frac{\nabla M_{\mathrm{A}} \times \boldsymbol{B}_{S}}{\sqrt{\left(\mu_{0} \rho\right)\left(1-M_{\mathrm{A}}^{2}\right)}}=\frac{M_{\mathrm{A}}}{\mu_{0}} \frac{\nabla \rho \times \boldsymbol{B}}{2 \rho^{3 / 2}} \\
& \frac{M_{\mathrm{A}}^{\prime}(A)\left(\nabla A \times \boldsymbol{B}_{S}\right)}{\sqrt{\left(\mu_{0} \rho\right)}\left(1-M_{\mathrm{A}}^{2}\right)^{3 / 2}}=\frac{\rho^{\prime}(A) M_{\mathrm{A}}(A)\left(\nabla A \times \boldsymbol{B}_{S}\right)}{2 \rho^{3 / 2} \sqrt{\mu_{0}}\left(1-M_{\mathrm{A}}^{2}\right)^{1 / 2}} \\
\Rightarrow & \frac{M_{\mathrm{A}}^{\prime}(A)}{M_{\mathrm{A}}(A)\left(1-M_{\mathrm{A}}^{2}(A)\right)}=\frac{\rho^{\prime}(A)}{\rho(A)} \\
\Rightarrow & \rho=\rho_{0}\left(\frac{M_{\mathrm{A}}^{2}}{1-M_{\mathrm{A}}^{2}}\right)^{1 / 2} .
\end{aligned}
$$

The density $\rho$ and the Alfvén Mach number are usually independently conserved quantities along the field lines, i.e. both $\rho=\rho(A)$ and $M_{\mathrm{A}}=M_{\mathrm{A}}(A)$ can be freely choosen. One recognizes that this cannot be fulfilled if the vorticity should vanish. Here, either the density is a specified function of the Alfvén Mach number, i.e. $\rho=\rho\left(M_{\mathrm{A}}(A)\right)$, or vice versa $M_{\mathrm{A}}=M_{\mathrm{A}}(\rho(A))$. If $\boldsymbol{\nabla} \times \boldsymbol{B}_{S} \neq \mathbf{0}$ then a solution is only possible if again relation Eq. (23) holds, i.e. the current must be perpendicular to the magnetic field. Concerning the current equation, Eq. (19) it can be recognized that this necessary condition Eq. (23) must also be fulfilled if one wants the steady-state-current to vanish. In the 2-D case, the current distribution and the magnetic field are then basically 1-D. This could, e.g. lead either to field lines being circles or straight field lines in 2-D.

In general (3-D), if $M_{\mathrm{A}}$ is non-constant and $\boldsymbol{B}_{S}$ is a nonpotential field, then the current can only vanish if $\boldsymbol{j}_{S \|}=\mathbf{0}$.

If $M_{\mathrm{A}}$ is constant, or its gradients can be neglected, and the corresponding magnetohydrostatic field is potential, it is possible to have no currents, or current sheet, but with the gradient term of the density it is possible to generate a vortex sheet in Eq. (24) at the same time. 


\subsection{Sequence of equilibria towards almost magnetohydrostatic configurations}

To analyse the connection between current and vortex sheets without loss of generality in 2-D, we search for an ansatz for the Alfvénic Mach number sheet. We use an ansatz where the Alfvén Mach number $M_{\mathrm{A}}$ is depending on one flux function, but basically this view can be transferred to the problem in 3-D with two flux functions, i.e. Euler potentials. We present the principle of how to construct a series of peaked function for the Alfvén Mach number, sharply separating regions with flow from regions with almost no flow $\left(M_{\mathrm{A}} \ll 1\right)$. The Alfvén Mach number should therefore be represented by a function series depending on a smallness parameter $\varepsilon \neq 0$, which tends to zero, to mimic an approximately static equilibrium. This has relevance for a lot of situations in which the complete or large part of the domain have a basically quasimagnetohydrostatic character, with some regions showing almost no flows along the field line, e.g. Neukirch (2005a) and Romeou and Neukirch (1999). The relevance for the case $M_{\mathrm{A}} \ll 1$ is valid especially in the case of a low $\beta$-plasma, as

$1 \gg \beta=\frac{p}{B^{2} /\left(2 \mu_{0}\right)}=\frac{p / \rho}{B^{2} /\left(2 \mu_{0} \rho\right)}=\frac{2}{\gamma} \frac{M_{\mathrm{A}}^{2}}{M_{S}^{2}}$

$\gamma$ being the polytropic exponent and $M_{S}$ the usual Mach number, i.e.

$\frac{p}{\rho^{\gamma}}=$ const $, \quad v_{S}^{2}=\frac{d p}{d \rho}, \quad M_{S}^{2}=\frac{v^{2}}{v_{S}^{2}}$.

Approximate incompressibility is plausible for Mach numbers $M_{S} \ll 1$, and hence the condition $M_{\mathrm{A}} \ll M_{S} \ll 1$, to guarantee a low $\beta$-plasma, is even more plausible. In fact, $M_{\mathrm{A}} \lesssim 1$ is possible for regions in which the plasma $\beta$ is much larger, e.g. in the vicinity of (degenerated) separatrices, being null sheets of the magnetic field, or regions where the magnetic field in general is weaker.

Taking a normalized flux function $A$ and a scaling factor $\varepsilon$ into account, a very general ansatz for $M_{\mathrm{A}}$ can be written as the following sequence of functions:

$$
\begin{aligned}
M_{\mathrm{A}} & = \pm\left|M_{\mathrm{A}, \max }\right| \varepsilon^{n}\left[\frac{ \pm 1+\tanh \left(\frac{A}{\varepsilon}\right)}{2}\right] \\
\lim _{\varepsilon \rightarrow 0} M_{\mathrm{A}} & =0 \\
\frac{d M_{\mathrm{A}}(A)}{d A} & \equiv M_{\mathrm{A}}^{\prime}(A)<\infty \\
\Rightarrow \quad M_{\mathrm{A}}^{\prime} & =\varepsilon^{n-1} \cosh ^{-2}\left(\frac{A}{\varepsilon}\right) \\
M_{\mathrm{A}} M_{\mathrm{A}}^{\prime} & =\varepsilon^{2 n-1}\left[\cosh ^{-2}\left(\frac{A}{\varepsilon}\right)+\frac{\sinh \left(\frac{A}{\varepsilon}\right)}{\cosh ^{3}\left(\frac{A}{\varepsilon}\right)}\right],
\end{aligned}
$$

i.e. the terms with $M_{\mathrm{A}}$ and $M_{\mathrm{A}}^{\prime}$ are bounded for $\varepsilon \neq 0$. A divergence of these terms and therefore the parametric formation of a vortex sheet can be recognized in the second row of the vortex equation, Eq. (24), if we consider $\lim \varepsilon \rightarrow 0$, and, of course, taking into account that $\nabla M_{\mathrm{A}}(A)=M_{\mathrm{A}}^{\prime}(A) \nabla A$. For small Alfvén Mach numbers, even if we do not only take potential fields, i.e. $\boldsymbol{\nabla} \times \boldsymbol{B}_{S}=\mathbf{0}$, into account, the dominant and important terms are that term in the vortex and current equations, which include at least the $\nabla M_{\mathrm{A}}$-term. We can distinguish different regimes for the exponent $n$, which are listed in the following:

1. For $n>1$, the current sheet and the vortex sheet contract with $1 / \varepsilon$ in Eq. (32), but their amplitudes converge to zero, as can be seen by taking the limit for Eq. (32) for $\varepsilon \rightarrow 0$. The reason is that the $M_{\mathrm{A}} M_{\mathrm{A}}^{\prime}$ term converges with $\varepsilon^{2 n-1}=\varepsilon^{2(1+|\delta|)-1}=\varepsilon^{1+|\delta|}$ to zero.

2. For $n=1$, the vortex sheet and the current contract, the amplitude of the vortex sheet stays finite, the current sheet amplitude converges to zero, also due to the $M_{\mathrm{A}} M_{\mathrm{A}}^{\prime}$-convergence, reading $\propto \varepsilon$.

3. In the range $1>n>\frac{1}{2}$, the vortex sheet and the current contract, the vortex sheet amplitude diverges with $\varepsilon^{-(1-n)}$ for $\varepsilon \rightarrow 0$, the amplitude of the current sheet converges to zero with $M_{\mathrm{A}} M_{\mathrm{A}}^{\prime} \propto \varepsilon^{|\delta|}$.

4. For $n=\frac{1}{2}$, the vortex sheet and the current contract, the vortex sheet diverges with $\varepsilon^{-\frac{1}{2}}$, the amplitude of the current sheet stays finite, converging with $M_{\mathrm{A}} M_{\mathrm{A}}^{\prime} \propto \varepsilon^{0}$.

5. For $0 \leq n<\frac{1}{2}$, the vortex sheet and the current sheet contract, the vortex sheet (with $\propto \varepsilon^{-2|\delta|}, \frac{1}{2} \geq|\delta|>0$ ) and the current sheet amplitude diverge $M_{\mathrm{A}} M_{\mathrm{A}}^{\prime} \propto$ $\varepsilon^{-\frac{1}{2}|\delta|}$; for $n=0$ the Alfvén Mach number stays finite and therefore cannot converge to a quasi static case. We therefore do not take values of $n<0$ into consideration.

These different cases imply that only for a special chosen funcion of the Alfvén Mach number, current and vortex sheets simultaneously appear, namely for the case of the exponent $n<1 / 2$. For the other cases it is at least necessary that $n<1$ to generate a singular vortex sheet in the limit $\varepsilon \rightarrow 0$, but without getting a current singularity. Case 5 guarantees the existence of both, a current sheet and a vortex sheet.

We did not discuss the influence of the $\nabla \rho$-term in the vortex-equation, Eq. (24), very intensively. The influence can also be very important, although it is with a $\propto M_{\mathrm{A}} M_{\mathrm{A}}^{\prime}-$ dependence of the same strength and quality as the dependence of the current density.

\subsection{General view on the connection between currents and vortices}

To clarify the structure of this connection between vortex sheets and current sheets from a different view, we introduce the abbreviations $\boldsymbol{G}_{\mathrm{A}}=\left(\nabla M_{\mathrm{A}} \times \boldsymbol{B}_{S}\right) /\left(1-M_{\mathrm{A}}^{2}\right)^{3 / 2}$ and 
$\boldsymbol{J}_{S}=\boldsymbol{j}_{S} /\left(1-M_{\mathrm{A}}^{2}\right)^{1 / 2}$ and can rewrite the vortex-equation Eq. (24) and the current-equation Eq. (19)

$$
\begin{aligned}
\boldsymbol{j} & =\frac{M_{\mathrm{A}}}{\mu_{0}} \boldsymbol{G}_{\mathrm{A}}+\boldsymbol{J}_{S} \\
\boldsymbol{\Omega} & =\frac{1}{\sqrt{\rho}} \boldsymbol{G}_{\mathrm{A}}+\frac{M_{\mathrm{A}}}{\mu_{0} \sqrt{\rho}} \boldsymbol{J}_{S}-\frac{M_{\mathrm{A}} \rho^{-\frac{3}{2}}}{2 \sqrt{\mu_{0}}} \nabla \rho \times \boldsymbol{B} \\
\Rightarrow \boldsymbol{\Omega} & =\frac{\rho^{-\frac{3}{2}}}{2} \frac{M_{\mathrm{A}}}{\mu_{0}} \nabla \rho \times \boldsymbol{B}+\frac{1}{\sqrt{\rho}} \boldsymbol{G}_{\mathrm{A}}+\frac{M_{\mathrm{A}}}{\mu_{0} \rho}\left(\boldsymbol{j}-\frac{M_{\mathrm{A}}}{\mu_{0}} \boldsymbol{G}_{\mathrm{A}}\right)
\end{aligned}
$$

The terms of order $O\left(M_{\mathrm{A}}^{2}\right)$ can be neglected for small values of $M_{\mathrm{A}}$. Then one can recognize that the most important term, the zeroth order in $M_{\mathrm{A}}$, is the $\boldsymbol{G}_{\mathrm{A}}$-term, followed by the gradient term of the density and the term with the current density as terms of first order in $M_{\mathrm{A}}$.

But even for the general case $M_{\mathrm{A}} \lesssim 1$, the $\boldsymbol{G}_{\mathrm{A}}$-term has the strongest influence on the generation of vorticity, as it scales only with $\rho^{-1 / 2}$ in contrast to the $\boldsymbol{j}$-term with a $1 / \rho$ dependence or the $\rho^{-3 / 2}$-dependence of the $\nabla \rho$-term. Only if the scale of the density gradient is much smaller than that of the Alfvén Mach number the gradient term of the density can dominate. The $\boldsymbol{G}_{\mathrm{A}}$-term is also favoured, as for values of $M_{\mathrm{A}}$ close to one the $\left(1-M_{\mathrm{A}}^{2}\right)^{3 / 2}$-dependence enhances the influence of the $\nabla M_{\mathrm{A}}$-term on the vorticity stronger than the $\left(1-M_{\mathrm{A}}^{2}\right)^{1 / 2}$-dependence of all other terms in the vorticity Eq. (35). The nature of this transformation technique shows that these steady-state current-vortex structures are related to static equilibria, and therefore the vortex structure and its scales governs the structure of the currents and current sheets.

\section{Examples}

As an example we investigate an initial 2-D-equilibrium in the form

$A(x, y)=x^{2}+\left(j_{\mathrm{z}} / 2-1\right) y^{2}$,

which reduces to a potential field solution for a vanishing current $j_{\mathrm{Z}}$ as shown in Fig. 1 panel $A(x, y)$. We describe a Mach-number-profile in the form

$M_{\mathrm{A}}(A)=\frac{M_{\text {Amax }}}{2}\left(1-\tanh \frac{A}{d}\right)$,

where $M_{\mathrm{Amax}}=0.5$ and $d=0.2$ are free parameters; see topleft panels 1 showing $M_{\mathrm{A}}(A)$ and $\frac{\partial M_{\mathrm{A}}(A)}{\partial A}$ for the profile and its derivative, respectively. This introduces a plasma flow on some field lines and a moderate flow gradient perpendicular to the x-point separatrices as shown in the top-right panels $M_{\mathrm{A}}$ and $\frac{\partial M_{\mathrm{A}}}{\partial A}$. As a consequence, the initial current free equilibrium develops a current and vortex sheet aligned with the separatrices (middle-right panels $J_{\mathrm{Z}}$ and $\Omega$, respectively). The bottom-right panel $\frac{J_{z}}{\Omega}$ shows the ratio of these quantities.
In Fig. 2 we use the same mathematical form, but provide a significantly steeper flow gradient $d=0.2 / 16$ along the separatrices, but a lower maximum flow velocity $M_{\mathrm{Amax}}=0.25$. The panels in Fig. 2 have the same meaning as in Fig. 1. We observe that both the current sheet and vortex sheet increase in magnitude, but the vortex sheet is more strongly affected, as the ratio $\frac{J_{z}}{\Omega}$ shows.

\section{Conclusions}

Numerical incompressible time dependent adaptive MHD simulations by Grauer and Marliani $(1998,2000)$ show that vortex sheets align with current sheets. Although it is not clear why this alignment occurs within the approach of these simulations, we could show that, for steady-state incompressible field-aligned flows, this alignment appears naturally in a non-trivial way: even if the Alfvén Mach number is non-constant, and non-trivial terms appear, resulting from the used non-canonical transformation mechanism, the resulting microscopic or thin structure and decrease of the amplitude of current and vortex strength show a clear alignment. The reason is that, in both representations of current and vorticity, the gradient of the the Alfvén Mach number $M_{\mathrm{A}}$ generates the thin structure of vortex and current sheet.

The larger the gradient, i.e. the smaller the lengthscale, the stronger the vorticity and the current. The difference is only that the current generating term is multiplied by $M_{\mathrm{A}}$, i.e. in the case of approximative magnetohydrostatics $M_{\mathrm{A}} \ll 1$, the vortex is stronger enhanced than the current. To obtain strong vortex sheets without strong current sheets, the plasma flow must change on significantly smaller scales than the magnetic field.

It is also noteworthy that the function $\rho$ can be chosen freely as a function of the Euler-potentials in the frame of this transformation technique. The free choice leads to strong influence of the vorticity on the density gradient. This may be important for regions across magnetic boundaries, e.g. helmet streamer regions, the heliopause or other astropauses, the magnetopause, etc., where inner and outer field lines and plasma regions are separated by separatrices or magnetopauses, separating regions of higher density from regions of lower density. Thus, it is very difficult to get current sheets without vortex sheets, as the used non-canonical transformation method automatically has to produce shear flows to create filamentary structured current sheets. These shear flows in fact imply vortex sheets, hence generating current sheets.

On the other hand, for special configurations it is also possible to create a current sheet with a vanishing vortex strength in 2-D, where one necessary condition is automatically fulfilled, namely that the parallel current with respect to the magnetic field vanishes, i.e. $\boldsymbol{j}_{\|}=\mathbf{0}$. But a special density distribution must also be included to be sufficient: normally the both free and independent integrals $M_{\mathrm{A}}$ and $\rho$ are then 
necessarily dependent functions of each other in 2-D, i.e. $\rho=\rho\left(M_{\mathrm{A}}(A)\right)$.

The velocity fields calculated from the quasi-static approach (see Schindler and Birn, 1987, and Wiechen and Schindler, 1988) show high bulk velocities parallel to the magnetic field, similar to the pure field-aligned flow of our presented calculations. There is also an analogy to the fine structures of current sheets in both approaches, namely the filamentary structure formation, i.e. the formation of a peaking, almost singular current, in time-dependent, quasi-static theory and the filamentary structure formation of the current sheet due to small-scale gradients of Alfvén Mach number of our stationary approach. The used technique to calculate exact solutions of ideal MHD from known magnetohydrostatic solutions is very important to understand the structure of current vortex sheets also regarding eruptive space plasma processes like magnetic reconnection and following magnetospheric substorms, eruptive flares or coronal mass ejections. Even if anomalous viscosity is damped or minimized (Ahmadi and Hirose, 1981) and shear flows in specific situations lower the reconnection rate (Karlicky, 1989), it is important to generate smaller-scale dissipation (see e.g. Baty et al., 2003).

"Flare observations should be used to investigate whether the large-scale vortical flows, required to sustain the maximum viscous dissipation rate, are indeed present in the flaring solar corona.", as theoretical investigations on steady reconnection and dissipation show (see Craig and Litvinenko, 2009).

Hence, we expect that in the vicinity of current sheets close to separatrices, it is possible to observe such strongly sheared flows. From that point of view, it would be interesting to search for small-scale shear flows in the vicinity of thin current sheets to compare this with initial configurations in active or eruptive regions in the solar atmosphere or in magnetospheres before magnetic (sub)storms. The involved scales may admittedly be extremely small.

It is also noteworthy that in adaptive mesh refinement analyses of magnetic reconnection, the "fractal" or better filamentary structure of current sheets can also be found and plays a very important role in fragmented reconnection, i.e. current-layer fragmentation (see, e.g. Bárta et al., 2011, and Bárta et al., 2010).

It would be of great interest to investigate steady-state current vortex sheets for general field-aligned flows.

Acknowledgements. D.H.N. acknowledges support from a visitor grant of the Max-Planck-Institute in Katlenburg-Lindau.

The service charges for this open access publication have been covered by the Max Planck Society.

Topical Editor I. A. Daglis thanks A. Anastasiadis and two other anonymous referees for their help in evaluating this paper.

\section{References}

Ahmadi, G. and Hirose, A.: Distortion of velocity gradient by anomalous viscosity, IEEE Transactions on Plasma Science, 9, 21-24, doi:10.1109/TPS.1981.4317375, 1981.

Bárta, M., Büchner, J., and Karlický, M.: Multi-scale MHD approach to the current sheet filamentation in solar coronal reconnection, Adv. Space Res., 45, 10-17, doi:10.1016/j.asr.2009.07.025, 2010.

Bárta, M., Büchner, J., Karlický, M., and Skála, J.: Spontaneous Current-layer Fragmentation and Cascading Reconnection in Solar Flares. I. Model and Analysis, Apj, 737, 24, doi:10.1088/0004-637X/737/1/24, 2011.

Baty, H., Keppens, R., and Comte, P.: The two-dimensional magnetohydrodynamic Kelvin-Helmholtz instability: Compressibility and large-scale coalescence effects, Phys. Plasmas, 10, 46614674, doi:10.1063/1.1624076, 2003.

Becker, U., Neukirch, T., and Schindler, K.: On the quasistatic development of thin current sheets in magnetotaillike magnetic fields, J. Geophys. Res., 106, 3811-3826, doi:10.1029/2000JA900141, 2001.

Craig, I. J. D. and Litvinenko, Y. E.: Anisotropic viscous dissipation in three-dimensional magnetic merging solutions, A\&A, 501, 755-760, doi:10.1051/0004-6361/200911784, 2009.

Eyink, G. L. and Aluie, H.: The breakdown of Alfvén's theorem in ideal plasma flows: Necessary conditions and physical conjectures, Physica D Nonlinear Phenomena, 223, 82-92, doi:10.1016/j.physd.2006.08.009, 2006.

Forbes, T. G. and Priest, E. R.: Photospheric Magnetic Field Evolution and Eruptive Flares, ApJ, 446, 377, doi:10.1086/175797, 1995.

Gebhardt, U. and Kiessling, M.: The structure of ideal magnetohydrodynamics with incompressible steady flow, Phys. Fluids B, 4, 1689-1701, doi:10.1063/1.860078, 1992.

Grauer, R. and Marliani, C.: Geometry of singular structures in magnetohydrodynamic flows, Phys. Plasmas, 5, 2544-2552, doi:10.1063/1.872939, 1998.

Grauer, R. and Marliani, C.: Current-Sheet Formation in 3D Ideal Incompressible Magnetohydrodynamics, Phys. Rev. Lett., 84, 4850-4853, doi:10.1103/PhysRevLett.84.4850, 2000.

Karlicky, M.: Effects of shear plasma flow on externally induced magnetic field line reconnection, Bulletin of the Astronomical Institutes of Czechoslovakia, 40, 23-27, 1989.

Neukirch, T.: Magnetic Field Extrapolation, in: Chromospheric and Coronal Magnetic Fields, edited by: Innes, D. E., Lagg, A., and Solanki, S. A., vol. 596 of ESA Special Publication, 2005a.

Neukirch, T.: Theory of Energy Storage and Release in the Solar Corona, in: The Dynamic Sun: Challenges for Theory and Observations, vol. 600 of ESA Special Publication, 2005b.

Nickeler, D. H. and Wiegelmann, T.: Thin current sheets caused by plasma flow gradients in space and astrophysical plasma, Ann. Geophys., 28, 1523-1532, doi:10.5194/angeo-28-15232010, 2010.

Nickeler, D. H., Goedbloed, J. P., and Fahr, H.-J.: Stationary fieldaligned MHD flows at astropauses and in astrotails. Principles of a counterflow configuration between a stellar wind and its interstellar medium wind, A\&A, 454, 797-810, doi:10.1051/00046361:20053062, 2006.

Petrie, G. J. D. and Neukirch, T.: Self-consistent Three-dimensional Steady-state Solutions of the MHD Equations with Field-aligned 
Incompressible Flow, Geophys. Astrophys. Fluid Dynamics, 91, 269-302, 1999.

Romeou, Z. and Neukirch, T.: Self-consistent Models of Solar Magnetic Structures in Three Dimensions, in: Magnetic Fields and Solar Processes, edited by: Wilson, A., vol. 448 of ESA Special Publication, 871-876, 1999.

Schindler, K. and Birn, J.: Self-consistent theory of time-dependent convection in the earth's magnetotail, J. Geophys. Res., 87, 2263-2275, doi:10.1029/JA087iA04p02263, 1982.

Schindler, K. and Birn, J.: On the generation of field-aligned plasma flow at the boundary of the plasma sheet, J. Geophys. Res., 92, 95-107, doi:10.1029/JA092iA01p00095, 1987.
Solanki, S. K., Lagg, A., Woch, J., Krupp, N., and Collados, M.: Three-dimensional magnetic field topology in a region of solar coronal heating, Nature, 425, 692-695, doi:10.1038/nature02035, 2003.

Wiechen, H. and Schindler, K.: Quasi-static theory of the earth's magnetotail, including the far tail, J. Geophys. Res., 93, 59225928, doi:10.1029/JA093iA06p05922, 1988.

Wiegelmann, T. and Schindler, K.: Formation of thin current sheets in a quasistatic magnetotail model, Geophys. Res. Lett., 22, 2057-2060, 1995. 